\title{
EVALUACIÓN DE MÉTODOS DE INJERTO EN SANDÍA (CITRULLUS LANATUS (THUNB.) MATSUM. \& NAKAI) SOBRE DIFERENTES PATRONES DE CALABAZA
}

\author{
EVALUATION OF GRAFTING METHODS IN WATERMELON \\ (CITRULLUS LANATUS (THUNB.) MATSUM. \& NAKAI) \\ ONTO DIFFERENT SQUASH ROOTSTOCKS
}

\author{
Jesús López-Elías ${ }^{1}$; Agustín R. Fco. Romo A. ${ }^{1}$, Joaquín G. Domínguez $S^{1}{ }^{1}$
}

\begin{abstract}
RESUMEN
Se llevó a cabo un experimento en un invernadero comercial para evaluar dos métodos de injerto en sandía (Citrullus lanatus (Thunb.) Matsum. \& Nakai), híbrido Tri-X 313 injertado sobre calabaza (Cucurbita maxima x Cucurbita moschata), híbridos interespecíficos RS1330, RS841, RS888 y RS1313. Esta técnica se está expandiendo considerablemente buscando reducir los daños causados por patógenos del suelo, incrementar la resistencia a la sequía, así como para mejorar la absorción de agua y nutrientes. Las plantas fueron injertadas utilizando las técnicas de aproximación y púa, siendo comparadas con plantas de sandía sin injertar. Se evaluaron la sobrevivencia, el número de hojas, la altura de planta y la relación diámetro del tallo. El experimento se desarrolló utilizando un diseño de bloques completos al azar, con cuatro repeticiones. El método de injerto usado no influyó en la sobrevivencia de la planta. El número de hojas y la altura de la planta fueron mayores en aquellas plantas injertadas mediante aproximación. La relación diámetro del tallo fue menor en aquellas plantas injertadas mediante aproximación.
\end{abstract}

Palabras clave: Injerto, patrón, calabaza, sandía.

\begin{abstract}
A trial was carried out in a commercial greenhouse to evaluate two grafting methods on watermelon (Citrullus lanatus (Thunb.) Matsum. \& Nakai), hybrid Tri-X Brand 313 grafted onto squash (Cucurbita maxima $x$ Cucurbita moschata), interespecific hybrids RS1330, RS841, RS888 and RS1313. This technique is now being expanded greatly in order to reduce infections caused by soil pathogens, to increase resistance to drought and to enhance water and nutrient uptake. Plants were grafted by tongue approach and cleft grafting, and compared with watermelon plants without grafting. Survival rate, leaf number, plant height and stem diameter relation were evaluated. The trial was carried out in a randomized complete block design, with four replications. Grafting methods had no influence on survival rate. Leaf number and plant height were greater in tongue approach grafted plants. Stem diameter relation was lower in tongue approach grafted plants.
\end{abstract}

Key words: Graft, rootstock, squash, watermelon.

\section{INTRODUCCIÓN}

La producción de hortalizas en México es una actividad importante del sector agrícola. Entre las 8 hortalizas más importantes, la sandía cubre una superficie de 38.393 ha con una producción de $24,4 \mathrm{t} \mathrm{ha}^{-1}$, ocupando Sonora el cuarto lugar con 2.966 ha y una producción de $29,1 \mathrm{t} \mathrm{ha}^{-1}$.
En el cultivo de hortalizas, como defensa contra diversos problemas bióticos (enfermedades del suelo y nematodos) y abióticos (déficit hídrico, encharcamiento, temperaturas extremas, salinidad, etc.), se plantea la práctica del injerto en variedades con alto potencial productivo. Su empleo incrementa la tolerancia de las plantas a los nematodos y las enfermedades del suelo, incrementa la resistencia a

1 Universidad de Sonora, Departamento de Agricultura y Ganadería. Carretera a Bahía de Kino, km. 21. Hermosillo, Sonora. México. E-mail: lopez_eliasj@guayacan.uson.mx

Fecha de Recepción: 01 Agosto 2006

Fecha de Aceptación: 05 Noviembre 2006 
la sequía y mejora la absorción de agua y nutrientes, cuyo resultado final es un mayor vigor en la planta, favoreciendo con ello el desarrollo de la agricultura sustentable del futuro.

A la par con el déficit de agua para riego por el que atraviesa el campo, el cultivo intensivo ha propiciado la presencia de enfermedades en los suelos agrícolas agravando la situación, considerándose por ello prioritario buscar nuevas técnicas de producción en el sector agrícola. Aunado a lo anterior, las restricciones en cuanto al uso de productos como el bromuro de metilo dan un incentivo al desarrollo e implementación de nuevas tecnologías que permitan al agricultor afrontar el problema de enfermedades presentes en el suelo, a saber de otras condiciones adversas, siendo el uso del injerto una técnica innovadora en nuestro país.

La producción de plantas injertadas se inició en Japón y Corea a fines de los años 20 con sandía (Citrullus lanatus (Thunb.) Matsum. \& Nakai) injertada sobre patrón de calabaza (Lee, 1994). Posteriormente, en los 50 se injertó berenjena (Solanum melongena L.) sobre berenjena (Solanum integrifolium Poir.). Desde entonces, la superficie de hortalizas que utiliza plantas injertadas se ha incrementado considerablemente (Oda, 1993).

En la actualidad, Japón y Corea son los principales países productores de plantas injertadas, con 750 y 540 millones de plantas al año, respectivamente, seguidos por España, con 154 millones de plantas injertadas al año, siendo sandía y tomate los principales cultivos que se injertan (Leonardi y Romano, 2004). En México esta técnica es relativamente reciente, llegándose a injertar poco más de 60 mil plantas de tomate, pimiento y sandía en los estados de Sinaloa y Jalisco (Burgueño y Barba, 2001).

El principal objetivo de las plantas injertadas ha sido lograr el control de enfermedades provocadas por organismos del suelo, tales como Fusarium sp., Verticillium sp. y Pyrenochaeta sp., haciendo uso de patrones tolerantes a dichos patógenos (Blancard et al., 1991; Messiaen et al., 1995). Con esta técnica se aprovecha la tolerancia del sistema radical del patrón y los caracteres productivos favorables de una variedad susceptible.

La resistencia de las plantas injertadas está condicionada tanto por el patrón como por la variedad (Muller y Li, 2002) y aunque el vigor de la planta injertada es intermedio entre el del patrón y la variedad, la influencia del primero es mayor.
Este incremento en el vigor, que generalmente proporciona el patrón sobre la variedad, permite utilizar un menor número de plantas por unidad de superficie (Miguel, 1997).

El objetivo principal del presente trabajo fue evaluar la técnica de injerto utilizando diferentes patrones de calabaza.

\section{MATERIALES Y MÉTODOS}

El trabajo de investigación se realizó en un invernadero comercial protegido con cubierta plástica, localizado en la Costa de Hermosillo (29 00' 52" latitud Norte, $111^{\circ} 07$ ' 56" longitud Oeste), durante los meses de diciembre de 2005 a febrero de 2006.

El diseño experimental fue de bloques completos al azar en arreglo factorial, con 10 tratamientos y 4 repeticiones. Se evaluaron dos métodos de injerto sobre 4 patrones de calabaza (Cucurbita maxima x Cucurbita moschata), híbridos interespecíficos RS1330, RS841, RS888 y RS1313 y el correspondiente testigo sin injertar. Cada repetición estaba constituida por 10 plantas.

La semilla de sandía (Citrullus lanatus (Thunb.) Matsum. \& Nakai), híbrido Tri-X 313, fue sembrada el día 27 de diciembre de 2005 en charolas de semillero de 128 cavidades, colocando una semilla por cavidad. La semilla del patrón se sembró el día 4 de enero de 2006 en charolas de semillero de 200 cavidades, colocando también una semilla por cavidad.

El injerto se realizó el día 22 de enero de 2006, siguiendo la técnica descrita por Oda (1995). En ambas técnicas de injerto se eliminó completamente el meristemo apical del patrón, dejando únicamente los cotiledones. Como material se sujeción se utilizaron pinzas para injertos.

Una vez realizado el injerto, las plantas injertadas se colocaron en vasos de poliestireno de 8 onzas, colocando una planta por vaso. En el caso del injerto de aproximación, a los 14 días después del injerto se procedió a separar la sandía del patrón, eliminando el hipocotilo de la sandía por debajo del injerto, al igual que la porción superior del patrón.

Se evaluaron la sobrevivencia de las plantas, el número de hojas por planta, la altura de planta y la relación diámetro del tallo. Esta última variable como resultado de dividir el diámetro del tallo del patrón sobre el diámetro del tallo de la sandía. 
La sobrevivencia y el número de hojas por planta se evaluaron a los 16 días de realizado el injerto. La altura de la planta y la relación diámetro del tallo se evaluaron cuando la mayoría de las plantas mostraban 4 hojas bien desarrolladas. En la técnica de aproximación, la evaluación se realizó a los 21 días posteriores al injerto, mientras que en el caso de la técnica de púa, la evaluación se realizó a los 31 días posteriores al injerto.

Los datos obtenidos se sometieron a análisis de varianza. En todos los casos, para la comparación de medias se usó la prueba de Duncan al 5\%.

\section{RESULTADOS Y DISCUSIÓN}

En el Cuadro 1 se presentan los valores obtenidos para la variable sobrevivencia de la planta. Comparando las técnicas de injerto, los resultados mostraron que no existen diferencias significativas entre ambas técnicas evaluadas. En lo que respecta a los patrones, en el injerto de aproximación no se obtuvieron diferencias significativas entre los patrones utilizados, mientras que en el caso del injerto de púa, la mayor sobrevivencia se obtuvo al utilizar los patrones RS1330 y RS841, sin diferencias significativas con respecto al testigo sin injertar, observándose una menor sobrevivencia al utilizar el patrón RS1313.

Resultados similares fueron obtenidos por Miguel (1997), Rojas y Riveros (2001) y LópezElías et al. (2005b), quienes comparando diferentes técnicas de injerto observaron que con la técnica de aproximación se obtuvo la mayor sobrevivencia, misma que resultó menor al utilizar la técnica de púa.

Entre columnas, medias seguidas por la misma letra minúscula no son significativamente diferentes $(P=0,05)$.

Entre hileras, medias seguidas por la misma letra mayúscula no son significativamente diferentes $(P=0,05)$.

En cuanto al número de hojas, a los 16 días de realizado el injerto (Cuadro 2), se observó una interacción significativa entre las técnicas de injerto evaluadas, en donde el injerto de aproximación mostró un mayor número de hojas debido principalmente a una menor deshidratación del injerto propiciado por la permanencia del sistema radical de ambas plantas, hasta completada la unión del injerto. En lo que respecta a los patrones, no se observaron diferencias significativas entre ellos.

Para la altura de la planta (Cuadro 3), la técnica de injerto influyó significativamente sobre dicha variable, siendo en aquella de aproximación donde se obtuvieron plantas con mayor altura, sin diferencias significativas entre los patrones evaluados. En el caso del injerto de púa, dicha variable presentó diferencias significativas entre los patrones utilizados, obteniéndose una menor altura de planta al utilizar el patrón RS1313.

Los resultados obtenidos concuerdan con aquellos de López et al. (2005a), quienes comparando diferentes técnicas de injerto en dos cultivares de

Cuadro 1

Sobrevivencia (\%) de plantas de sandía usando dos técnicas de injerto, aproximación y púa, a los 16 días de realizado el injerto

\begin{tabular}{|c|c|c|c|}
\hline Patrón & Aproximación & Púa & Promedio \\
\hline RS1330 & A $93,3 \mathrm{~b}$ & A $96,7 \mathrm{ab}$ & 95,0 \\
\hline RS841 & A $90,0 \mathrm{~b}$ & A $93,3 \mathrm{ab}$ & 91,6 \\
\hline RS888 & A $86,7 \mathrm{~b}$ & A $86,7 \mathrm{bc}$ & 86,7 \\
\hline RS1313 & A $90,0 \mathrm{~b}$ & A $76,7 \mathrm{c}$ & 83,3 \\
\hline Testigo & $100,0 \mathrm{a}$ & $100,0 \mathrm{a}$ & 100,0 \\
\hline cv $(\%)$ & $4,0^{*}$ & $7,5^{*}$ & \\
\cline { 1 - 3 }
\end{tabular}

* y n.s. representan significancia al 5\% y no significancia estadística, respectivamente.

Entre columnas, medias seguidas por la misma letra minúscula no son significativamente diferentes $(P=0.05)$.

Entre hileras, medias seguidas por la misma letra mayúscula no son significativamente diferentes $(P=0.05)$. 
Cuadro 2

Número de hojas en sandía usando dos técnicas de injerto, aproximación y púa, a los 16 días de realizado el injerto

\begin{tabular}{|c|c|c|c|}
\hline Patrón & Aproximación & Púa & Promedio \\
\hline RS1330 & A 3,2 a & B 1,6 b & 2,4 \\
\hline RS841 & A 3,6 a & B $1,7 \mathrm{~b}$ & 2,6 \\
\hline RS888 & A 3,5 a & B $1,9 \mathrm{~b}$ & 2,7 \\
\hline RS1313 & A 3,6 a & B $1,8 \mathrm{~b}$ & 2,7 \\
\hline Testigo & $3,3 \mathrm{a}$ & $3,3 \mathrm{a}$ & 3,3 \\
\hline cv $(\%)$ & 7,4 n.s. & $16,4 * *$ & \\
\hline
\end{tabular}

*** representan significancia al $1 \%$

n.s. no significancia estadística, respectivamente.

Entre columnas, medias seguidas por la misma letra minúscula no son significativamente diferentes $(P=0.05)$.

Entre hileras, medias seguidas por la misma letra mayúscula no son significativamente diferentes $(P=0.05)$.

\section{Cuadro 3}

Altura de la planta (cm) de sandía usando dos técnicas de injerto, aproximación y púa, a los 21 y 31 días de realizado el injerto, respectivamente

\begin{tabular}{|c|c|c|c|}
\hline Patrón & Aproximación & Púa & Promedio \\
\hline RS1330 & A $12,0 \mathrm{~b}$ & A 10,5 b & 11,2 \\
\hline RS841 & A $11,4 \mathrm{~b}$ & B $9,5 \mathrm{bc}$ & 10,4 \\
\hline RS888 & A $11,9 \mathrm{~b}$ & B 9,1 bc & 10,5 \\
\hline RS1313 & A $12,0 \mathrm{~b}$ & B $7,0 \mathrm{c}$ & 9,5 \\
\hline Testigo & $14,5 \mathrm{a}$ & $14,5 \mathrm{a}$ & 14,5 \\
\hline $\mathrm{cv}(\%)$ & $7,2^{*}$ & $13,3^{* *}$ & \\
\hline
\end{tabular}

${ }^{*} \mathrm{y}{ }^{* *}$ representan significancia al 5 y $1 \%$, respectivamente.

Entre columnas, medias seguidas por la misma letra minúscula no son significativamente diferentes $(P=0.05)$.

Entre hileras, medias seguidas por la misma letra mayúscula no son significativamente diferentes $(P=0.05)$.

sandía observaron que con la técnica de aproximación se obtuvo una mayor altura de planta.

La menor altura de planta que se observa en el injerto de púa se debe principalmente al retraso en el crecimiento que se tiene con dicha técnica, propiciado por la eliminación del sistema radical de la sandía al momento de realizar el injerto y a las condiciones ambientales prevalecientes durante el proceso de unión y aclimatación de las plantas injertadas.

Para la relación diámetro del tallo, como se observa en el cuadro 4, la técnica de injerto influyó sobre dicha variable, siendo en aquella de aproximación en el caso de los patrones RS1330 y RS888 donde se obtuvieron diferencias significativas, con una menor relación diámetro de tallo con respecto al injerto de púa. En lo que respecta a los patrones, se obtuvieron diferencias significativas observándose una mayor relación diámetro del tallo en el patrón RS888, para ambas técnicas de injerto evaluadas.

La diferencia en la relación diámetro del tallo está asociada a la diferencia en vigor de los patrones utilizados, al igual que a la diferencia en el crecimiento de la planta de sandía propiciado por la técnica de injerto 


\section{Cuadro 4}

Relación diámetro del tallo en plantas de sandía usando dos técnicas de injerto, aproximación y púa, a los 21 y 31 días de realizado el injerto respectivamente

\begin{tabular}{|c|c|c|c|}
\hline Patrón & Aproximación & Púa & Promedio \\
\hline RS1330 & B $1,0 \mathrm{c}$ & A $1,2 \mathrm{ab}$ & 1,1 \\
\hline RS841 & A $1,1 \mathrm{ab}$ & A $1,2 \mathrm{ab}$ & 1,1 \\
\hline RS888 & B $1,2 \mathrm{a}$ & A $1,5 \mathrm{a}$ & 1,3 \\
\hline RS1313 & A $1,1 \mathrm{~b}$ & A $1,4 \mathrm{ab}$ & 1,2 \\
\hline Testigo & $1,0 \mathrm{c}$ & $1,0 \mathrm{~b}$ & 1,0 \\
\hline cv $(\%)$ & $4,7^{* *}$ & $17,1^{*}$ & \\
\hline
\end{tabular}

$*$ y ** representan significancia al 5 y $1 \%$, respectivamente.

Entre columnas, medias seguidas por la misma letra minúscula no son significativamente diferentes $(P=0.05)$.

Entre hileras, medias seguidas por la misma letra mayúscula no son significativamente diferentes $(P=0.05)$.

utilizada, observándose un retraso en el crecimiento con el injerto de púa que repercutió directamente en el índice de área foliar (datos no presentados).

Los resultados obtenidos vienen a reforzar lo mencionado por González et al. (2003), en el sentido de que el diámetro del tallo del patrón es un factor determinante en el éxito de los injertos, asociado ello a la regeneración de haces vasculares.

\section{CONCLUSIONES}

La sobrevivencia de las plantas es independiente de la técnica de injerto y está asociada al patrón utilizado, observándose la mayor sobrevivencia en los patrones RS1330 y RS841.

\section{LITERATURA CITADA}

BLANCARD, D., H. LECOQ Y M. PITRAT. 1991. Enfermedades de las cucurbitáceas: observar, identificar, luchar. 301 p. Mundi-Prensa, Madrid, España.

BURGUEÑO, H. Y M. BARBA. 2001. El injerto en hortalizas. Hortalizas, Frutas y Flores. Ed. Agro Síntesis. México. 31: 8-13.

GONZÁLEZ, J.M., F. RADILLO, F. DE J. MARTÍNEZ Y M. BAZÁN. 2003. Evaluación de diferentes portainjertos en el desarrollo vegetativo del cultivo de la sandía (Citrullus lanatus) variedad Tri-x 313. Memorias del X Congreso Nacional de la Sociedad de Ciencias Hortícolas. México. p. 43.

LEE, J. 1994. Cultivation of grafted vegetables I. Current status, grafting methods, and benefits. HortScience 29: 235-239.
El número de hojas en las plantas injertadas varía con la técnica de injerto, obteniéndose un mayor número de hojas en el injerto de aproximación, independientemente del patrón utilizado. Por lo anterior, la implementación de la técnica de injerto de púa trajo consigo un retraso de 10 días para el trasplante de la planta en campo.

La altura de las plantas injertadas varía con la técnica de injerto, obteniéndose una mayor altura de planta en el injerto de aproximación, independientemente del patrón utilizado.

La relación diámetro del tallo es dependiente tanto de la técnica de injerto como del patrón utilizado, obteniéndose una menor relación diámetro del tallo en el injerto de aproximación, con el patrón RS1330.

LEONARDI, C. AND D. ROMANO. 2004. Recent issues on vegetable grafting. Acta Hort. 631: 163-174.

LÓPEZ-ELÍAS, J., A. ROMO Y E. ZAMORA. 2005a. Evaluación del uso del injerto en la propagación de sandía. Memorias del VIII Congreso Internacional en Ciencias Agrícolas. UABC. Mexicali, B.C. México. p. 256-259.

LÓPEZ-ELÍAS, J., J. ROMO, E. ZAMORA Y D.R. FERNÁNDEZ. 2005b. Evaluación de diferentes técnicas de injerto en sandía. Memorias del XI Congreso Nacional de la Sociedad Mexicana de Ciencias Hortícolas. Chihuahua, Chih. México. p. 225-227.

MESSIAEN, C., D. BLANCARD, F. ROUXEL Y R. LAFON. 1995. Enfermedades de las hortalizas. 576 p. Mundi-Prensa, Madrid, España. 
MIGUEL, A. 1997. Injerto de hortalizas. 88 p. Generalitat Valenciana. Conselleria de Agricultura, Pesca y Alimentación, Valencia, España.

MULLER, D.S. AND S. LI. 2002. Use of aeroponic chambers and grafting to study partial resistance to Fusarium solani f. sp. Glycines in soybean. Plant Disease 86: 1223-1226.
ODA, M. 1993. Present state of vegetable production using grafted plants in Japan. Agr. Hort. 68: 442-446.

ODA, M. 1995. New grafting methods for fruit-bearing vegetables in Japan. Japan Agricultural Res. Qrty. 29: 187-194.

ROJAS, L. Y F. RIVEROS. 2001. Efecto del método y edad de las plántulas sobre el prendimiento y desarrollo de injertos de melón (Cucumis melo). Agricultura Técnica 61: 262-274. 